\title{
Accidental Death
}

National Cancer Institute

\section{Source}

National Cancer Institute. Accidental Death. NCI Thesaurus. Code C90351.

An indication that the subject's death was due to a mishap. 